DOI: 10.15593/RZhBiomeh/2016.3.04

УДК 531/534:[57+61]

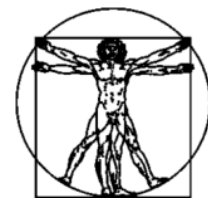

Росеийекий Журнал Биомеханики www.biomech.ru

\title{
КОЭФФИЦИЕНТЫ ПРОДОЛЬНОЙ СТАБИЛЬНОСТИ ДЕНТАЛЬНЫХ ИМПЛАНТАТОВ
}

\author{
В.А. Ерошин ${ }^{1}$, М.В. Джалалова ${ }^{1}$, А.В. Бойко ${ }^{1}$, С.Д. Арутюнов ${ }^{2}$, А.Г. Степанов ${ }^{2}$ \\ ${ }^{1}$ Научно-исследовательский институт механики МГУ имени М.В. Ломоносова, Россия, 119192, Москва, \\ Мичуринский проспект, 1, e-mail: margarita-vd@mail.ru \\ 2 Московский государственный медико-стоматологический университет имени А.И. Евдокимова, 127473, \\ Москва, ул. Делегатская, 20/1, e-mail: stepanovmd@list.ru
}

\begin{abstract}
Аннотация. Вводится понятие продольной стабильности дентальных имплантатов. Известно, что прибор Osstell ISQ (Implant Stability Quotient, Швеция) наряду с вынужденными колебаниями дентальных имплантатов, возникающими под действием поперечных нагрузок, может возбуждать продольные колебания и регистрировать их резонансную частоту. Цель работы - обоснование возможности использования коэффициентов продольной стабильности для оценки готовности дентальных имплантатов к функциональным нагрузкам, а также верификация методики их измерения с помощью прибора Osstell ISQ путем сравнения с соответствующими коэффрициентами продольной жесткости, измеряемыми методом лазерного тестирования. По сравнению с традиционной предлагаемая методика обладает рядом преимуществ и позволяет создать новую, более совершенную базу для неинвазивного мониторинга процесса остеоинтеграции дентальных имплантатов. Например, использование модифицированных «магнитных заглушек» вместо магнитных штифтов (SmartPeg) позволяет проводить измерения коэффициентов продольной стабильности через слизистую оболочку альвеолярной кости, т.е. не раскрывая дентальный имплантат для дальнейшего использования как опору под несъемный зубной протез, а модифицированные «магнитные колпачки» дают возможность определять коэффициенты продольной стабильности мини-имплантатов со сферической головкой, используемых для дополнительной фиксации съемных зубных протезов при полном отсутствии зубов. Необходимо подчеркнуть, что традиционная методика Osstell ISQ не позволяет проводить измерения коэффициентов стабильности указанных мини-имплантатов. Введение коэфффициентов продольной стабильности позволило построить простую теоретическую модель колебаний дентальных имплантатов, закрепленных в упругой и вязкоупругой среде, установить связь между коэффициентами продольной стабильности и продольной жесткости, дать оценку несущей способности дентальных имплантатов. Более того, при оценке их готовности к функциональным нагрузкам с помощью коэффициентов продольной стабильности рекомендации остаются прежними, так как отношение коэффрициентов стабильности при продольных и поперечных нагрузках должно быть близко к единице.
\end{abstract}

Ключевые слова: ортопедическая стоматология, дентальный имплантат, дентальный мини-имплантат, коэффициент стабильности, коэффрициент жесткости.

\footnotetext{
(C) Ерошин В.А., Джалалова М.В., Бойко А.В., Арутюнов С.Д., Степанов А.Г., 2016

Ерошин Владимир Андреевич, д.ф.-м.н., профессор, в.н.с. НИИ механики, Москва

Джалалова Маргарита Васильевна, к.ф.-м.н., с.н.с. НИИ механики, Москва

Бойко Андрей Владимирович, ведущий инженер лаборатории нестационарной гидродинамики, Москва

Арутюнов Сергей Дарчоевич, д.мед.н., профессор, завкафедрой клинической стоматологии № 2, Москва

Степанов Александр Геннадьевич, к.мед.н., доцент кафедры клинической стоматологии № 2, Москва
} 


\section{ВВЕДЕНИЕ}

В последние годы резонансно-частотный анализ стал одним из основных (механических) критериев готовности дентальных имплантатов к функциональным нагрузкам. Надежная оценка уровня остеоинтеграции имплантатов имеет решающее значение при выборе конструкции протеза, тактики функционального нагружения, а также при прогнозировании исхода ортопедического лечения. В этом смысле расширение возможностей прибора Osstell ISQ (Implant Stability Quotient) путем использования коэффициентов продольной стабильности $\left(\right.$ КСИ $\left.{ }_{b}\right)$ трудно переоценить.

Возможность использования прибора Osstell mentor в режиме возбуждения продольных колебаний дентальных имплантатов и регистрации соответствующих резонансных частот была отмечена во время командировки в Гетеборг (Швеция) в 2008 г., где проводились измерения коэффициентов стабильности дентальных имплантатов под руководством сотрудников фирмы Osstell AB, причем они не

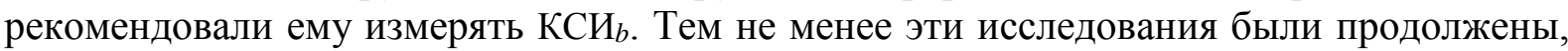
была установлена связь между коэффициентами продольной стабильности КСИ ${ }_{b}$ и продольной жесткости $K_{b}$, а изучение самого направления оказалось очень плодотворным. В дальнейшем нам придется иметь дело с несколькими коэффициентами стабильности и жесткости, поэтому введем следующие обозначения: при поперечных нагрузках на дентальный имплантат, направленных вдоль зубного ряда, для коэффициентов стабильности и жесткости будем использовать индекс $\tau$ $\left(К С И_{\tau}, K_{\tau}\right)$, поперек - индекс $n\left(К И_{n}, K_{n}\right)$ и вдоль оси имплантатов - индекс $b$ $\left(\mathrm{KC}_{b}, K_{b}\right)$.

Впервые сравнение коэффициентов поперечной стабильности (КСИ $)$ дентальных имплантатов, измеренных с помощью прибора Osstell mentor (Швеция), с коэффициентами поперечной жесткости $K_{n}$, измеренными по методике лазерного тестирования (Институт механики МГУ), было проведено в 2008 г., причем бо́льшая часть измерений КСИ ${ }_{n}$ была проведена в Гетеборге шведскими операторами [4]. Это исследование показало, что показания прибора Osstell mentor хорошо согласуются со значениями коэффициентов жесткости $(55<$ КСИ < 85). Однако при уменьшении жесткости крепления дентального имплантата в аналогах костной ткани разброс измерений значительно увеличивался, причем асимптотика поведения коэффициентов стабильности КСИ при стремлении коэффициентов жесткости к нулю $\left(K_{n} \rightarrow 0\right)$ была неверной. В новой версии прибора Osstell ISQ эти недостатки были устранены.

Большая серия экспериментов, проведенная авторами с использованием четырех приборов версии Osstell ISQ, показала верную асимптотику (при малых значениях коэффициентов жесткости коэффициенты стабильности также были малыми). Необходимо отметить, что при значениях коэффициента поперечной жесткости $K_{n}>0,5 \mathrm{MH} / \mathrm{M}$ (коэффициенты поперечной стабильности КСИ $>$ (50) результаты измерений, полученные при использовании двух различных версий прибора, практически не отличались (различие было в пределах точности измерений).

В данной работе исследуется последняя версия прибора Osstell ISQ и проведена его верификация путем сравнения коэффициентов продольной стабильности КСИ $\mathrm{c}_{b}$ коэффициентами продольной жесткости $K_{b}$, а также описаны новые по сравнению с традиционной методикой измерения возможности прибора.

\section{МАТЕРИАЛЫ, ПРИБОРЫ И МЕТОДЫ ИЗМЕРЕНИЯ}

При проведении исследований использовались классические винтовые дентальные имплантаты фирмы Conmet (Россия) и мини-имплантаты (MDI) фирмы $3 M E S P E$ (США), закрепленные в аналогах костной ткани из твердого пенопласта, 
полиуретана, липы и боксила. Форма имплантатов, а также их размеры и масса приведены в работе [5].

Коэффициент продольной жесткости $K_{b}$ измерялся методом лазерного тестирования и введен как отношение продольной силы $F$ к соответствующему поступательному перемещению имплантата вдоль оси симметрии:

$$
K_{b}=F / \Delta \text {, }
$$

где $F$ - сила, действующая вдоль оси имплантата; $\Delta$ - его перемещение, мкм (коэффициент поперечной жесткости определяется аналогично: $K_{n}=F / \Delta$, только в этом случае $F$ - поперечная сила и $\Delta$ - перемещение точки приложения нагрузки к дентальному имплантату).

Будем предполагать, что имплантаты являются абсолютно жесткими, а их перемещения происходят только за счет упругих свойств костной ткани. На рис. 1 изображена схема измерения коэффициентов продольной жесткости, где 1 - винтовой имплантат, 2 - аналог костной ткани. Штифт 3, опирающийся на дентальный имплантат, нагружается силой $F$. Он жестко соединен с рычагом 4, вращающимся вокруг оси, проходящей через точку $O$. При этом точка падения луча лазера 5 , закрепленного на рычаге $O A$, совершает перемещение $\xi$ на экране 6.

Величина угла поворота $\varphi$ рычага $O A$ и вертикальное перемещение имплантата $\Delta$ определяются по формулам $\varphi=\xi / L, \Delta=\varphi \cdot O A$, где $L(15 \leq L \leq 50$ м $)$ - расстояние от центра вращения рычага $O$ до экрана $(\xi<<L)$. Нагружение проводилось грузиками таким образом, чтобы линия действия силы проходила вдоль оси имплантата (при растягивающих нагрузках использовался блок). Подробно методика измерения описана в работе [4].

Для измерения коэффициентов продольной стабильности выбирали приборы Osstell ISQ. Они удобны, атравматичны и могут применяться с момента установки имплантатов. Процедура измерения коэффициентов поперечной стабильности при использовании стандартных штифтов (SmartPeg) такова, что каждый раз для этого необходимо хирургическим способом открывать слизисто-надкостничный лоскут,

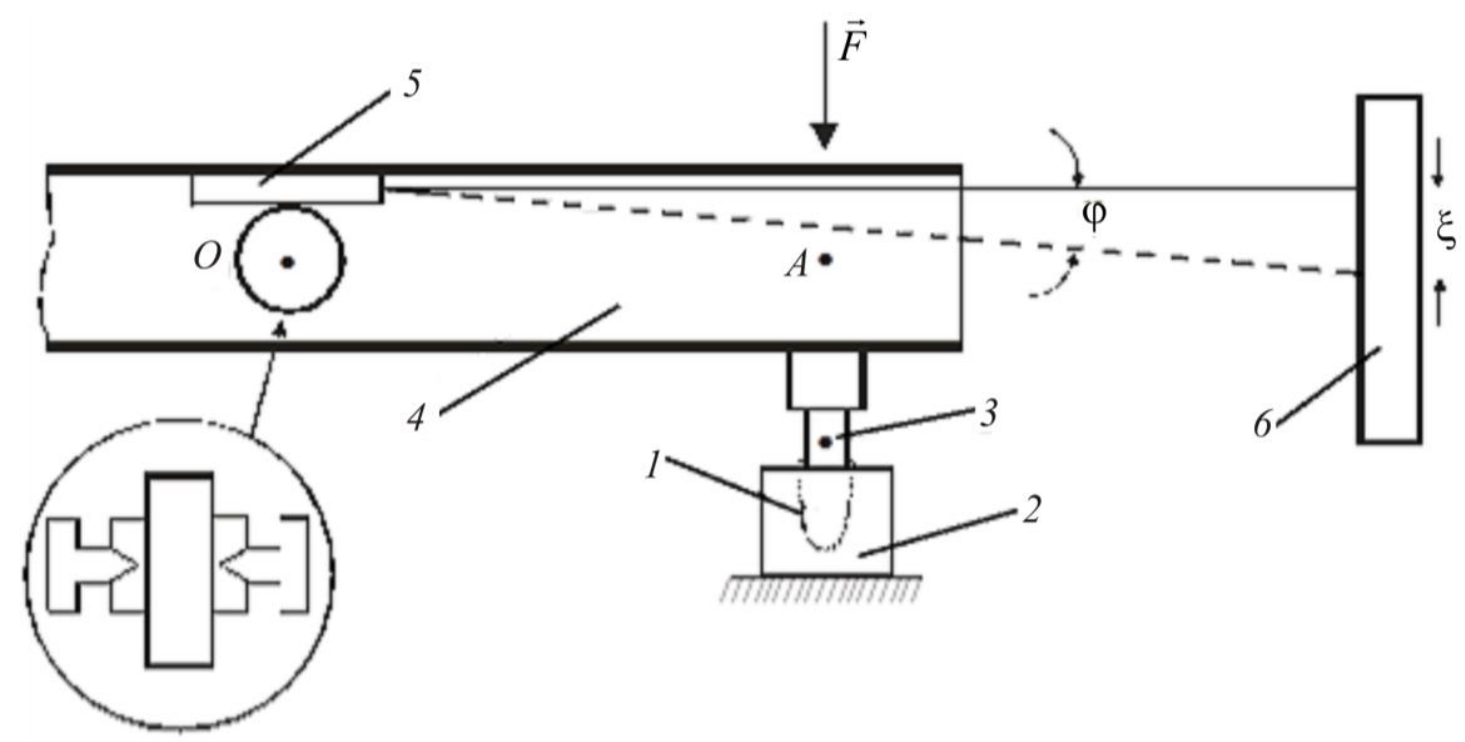

Рис. 1. Схема установки для определения коэффициентов продольной жесткости: 1 - имплантат; 2 - аналог костной ткани; 3 - штифт; 4 - рычаг-коромысло;

$$
5 \text { - лазер; } 6 \text { - экран }
$$


отвинчивать заглушку, закрывающую резьбовое отверстие в имплантате (в которое потом крепится несущая часть протеза), и устанавливать магнитный штифт, являющийся элементом измерительной системы прибора. Затем проводится измерение $K C И_{n}$ и опять ушивается слизисто-надкостничный лоскут, т.е. слежение за динамикой остеоинтеграции возможно. Однако оно не только требует определенных затрат сил и времени, но и сопряжено с заметными нагрузками на еще не окрепшую костную ткань. Это вмешательство может негативно отразиться на процессе остеоинтеграции.

Тем не менее если вместо обычной заглушки, которая закрывает резьбовое отверстие имплантата в процессе остеоинтеграции, использовать магнитную заглушку с тем же самым магнитом, что и в магнитном штифте (рис. 2), подобных проблем не возникает, так как измерение КСИ $b$ можно будет проводить через слизистую оболочку альвеолы кости без какой-либо специальной подготовки. Отметим также, что для возбуждения продольных колебаний не нужен магнитный штифт такой большой длины. Основное преимущество магнитной заглушки состоит в том, что процедура измерения значительно упрощается и появляется возможность проводить мониторинг процесса остеоинтеграции, не вскрывая слизисто-надкостничный лоскут.

В настоящее время широкое признание во всем мире, в том числе и в России, получила методика протезирования с использованием дентальных мини-имплантатов (Mini Dental Implants). Съемные зубные протезы массово применяются при лечении большинства больных пожилого возраста с полным отсутствием зубов. Однако их фиксация и стабилизация представляют определенные сложности, что, прежде всего, связано с неблагоприятными клиническими условиями протезного ложа, в особенности на нижней челюсти.

Использование дентальных мини-имплантатов в качестве дополнительных удерживающих элементов съемных зубных протезов позволяет если не исключить указанные недостатки, то, по крайней мере, в значительной степени их нивелировать.

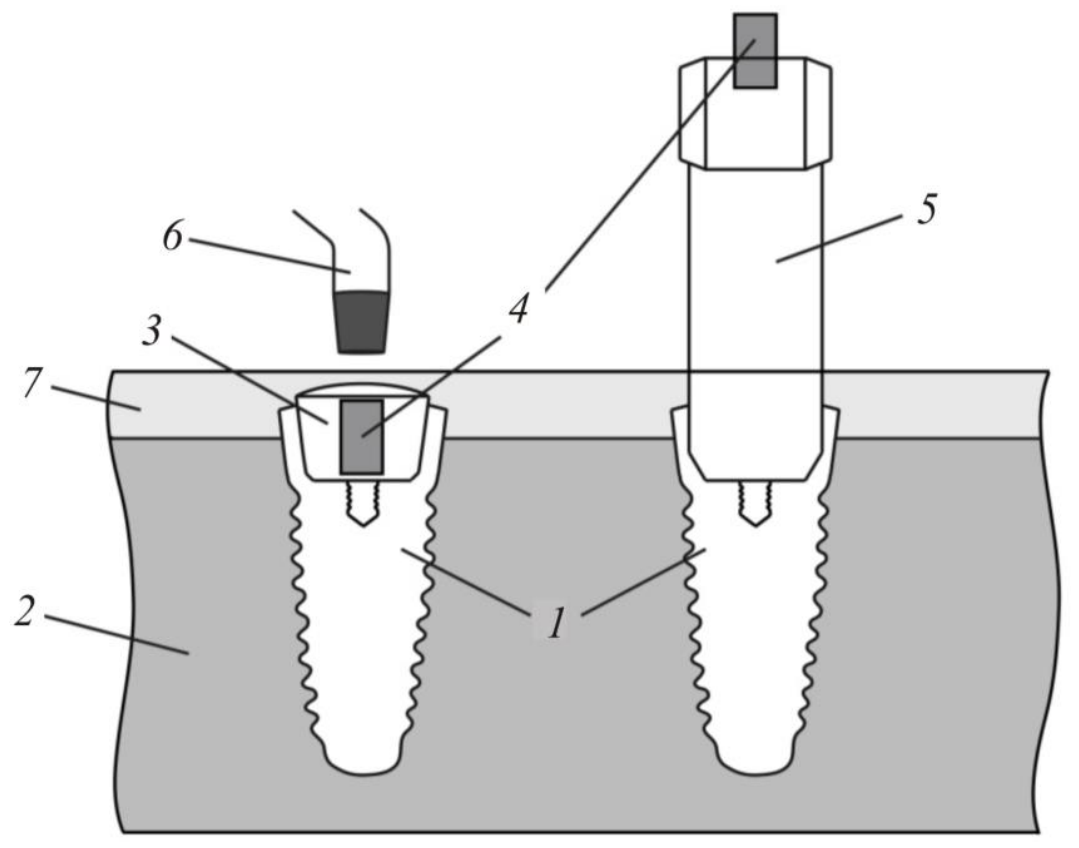

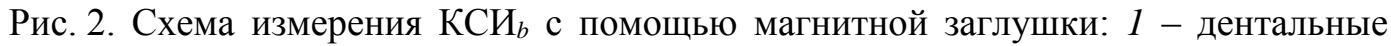
имплантаты; 2 - костная ткань; 3 - заглушка с магнитом 4; 5 - штифт SmartPeg; 6 - измерительный зонд Osstell ISQ; 7 - слизистая оболочка) 


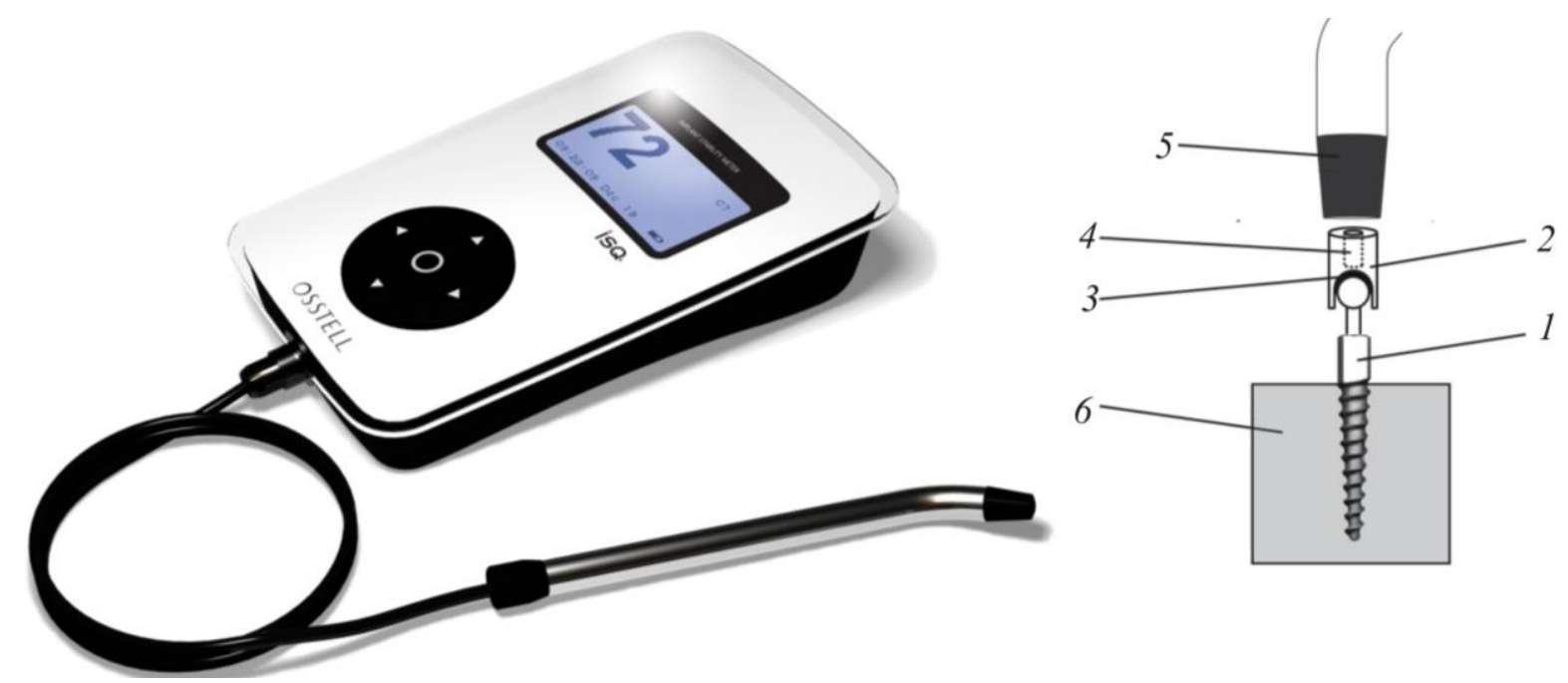

Рис. 3. Схема измерения коэффициентов продольной стабильности $M D I$ с помощью прибора Osstell ISQ: 1 - мини-имплантат; 2 - измерительный колпачок; 3 - слой временного цемента; 4 - магнитный элемент измерительного колпачка; 5 - зонд прибора Osstell ISQ; 6 - костная ткань

По сравнению с классическими винтовыми имплантатами мини-имплантаты обладают рядом преимуществ: для их установки значительно меньше противопоказаний, ниже требования к качеству костной ткани и геометрии гребня альвеолярной части нижней челюсти из-за их сравнительно небольшого диаметра, установка производится за одно посещение при минимальном хирургическом вмешательстве. Использование мини-имплантатов значительно дешевле, чем применение традиционных дентальных имплантатов [13-15].

Популярность и широкое использование Mini Dental Implants, естественно, привели к изменению их геометрии и расширению функционального назначения [10-12]. Если первоначально они применялись исключительно для улучшения фиксации и стабилизации съемных протезов при полном отсутствии зубов, то постепенно при увеличении размеров Mini Dental Implants появились попытки их использования в качестве несущих элементов при небольших вертикальных нагрузках (отсутствие одного зуба).

После установки мини-имплантатов также необходимо проводить мониторинг процесса остеоинтеграции, причем особенно важно следить за динамикой изменения их подвижности в первые недели после установки для того, чтобы проводить коррекцию и регулировать нагрузки. Методика оценки подвижности Mini Dental Implants с помощью прибора Osstell ISQ, предложенная нами, позволяет проводить мониторинг процесса остеоинтеграции [1]. Сущность ее состоит в следующем. На сферической головке Mini Dental Implants с помощью стоматологического цемента для временной фиксации несъемных ортопедических конструкций крепится специально разработанный измерительный колпачок $\mathrm{c}$ магнитным элементом, позволяющий определять коэффициенты стабильности при приложении к нему продольных и поперечных периодических нагрузок (рис. 3).

Эксперименты показали, что значения коэффициентов КСИ $\mathrm{K}_{b}$ продольной стабильности дентальных имплантатов, закрепленных в аналогах костной ткани (см. рис. 2), измеренные прибором Osstell mentor при использовании стандартного магнитного штифта, практически совпадают с соответствующими значениями

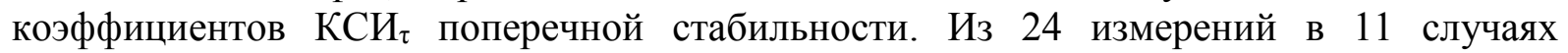
отношение $\mathrm{KCИ}_{b} / \mathrm{KCИ}_{\tau}$ было в точности равно единице, в остальных 13 различие составляло менее $7 \%$. 


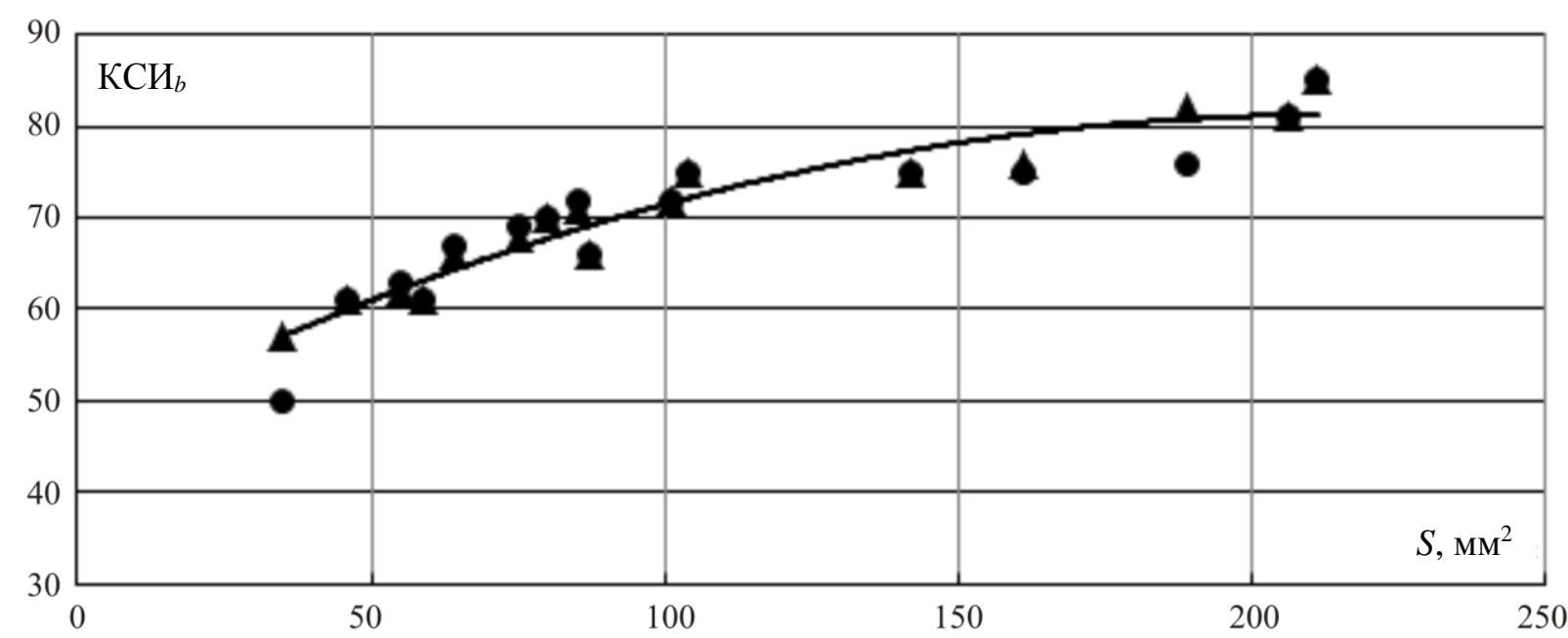

Рис. 4. Зависимость коэффициента продольной стабильности от площади контакта дентального имплантата с аналогом костной ткани из твердого пенопласта: $\boldsymbol{\Delta}-$ без покрытия, •-c покрытием

Более того, измерения, проведенные в последнее время приборами Osstell ISQ для дентальных имплантатов и Mini Dental Implants, закрепленных в аналогах костной ткани из полиуретана и липы, при использовании магнитных заглушек и магнитных колпачков показали еще более высокий уровень совпадения значений КСИ $\mathrm{K}_{b} \mathrm{KCИ}_{\tau}$ : из 1000 измерений для дентальных имплантатов и Mini Dental Implants различных размеров $85 \%$ дали точное совпадение $\left(К С И_{b}=\mathrm{KCИ}_{\tau}\right)$, и лишь в $15 \%$ случаях различие составляло около $5 \%$. Таким образом, коэффициент продольной стабильности с тем же успехом может использоваться для оценки готовности имплантатов к функциональным нагрузкам. Здесь необходимо отметить, что в отличие от отношения КСИ $\mathrm{K}_{b} / \mathrm{K}_{\tau}$ отношение $\mathrm{KCИ}_{n} / К С И_{\tau}$ (т.е. поперек и вдоль зубного ряда) зависит от размеров альвеолярного гребня и может быть заметно меньше единицы.

\section{ИЗМЕРЕНИЕ КОЭФФИЦИЕНТОВ ПРОДОЛЬНОЙ СТАБИЛЬНОСТИ. МАГНИТНАЯ ЗАГЛУШКА И МАГНИТНЫЙ КОЛПАЧОК}

Приведем опытные данные, показывающие возможность использования магнитной заглушки для неинвазивного мониторинга процесса остеоинтеграции. Для этого была проведена серия экспериментов с имплантатами, в которых были установлены магнитные заглушки. В качестве аналога костной ткани использовался твердый пенопласт. Рассматривались два варианта крепления магнита в имплантате. В первом случае магнит цилиндрической формы и размерами $d=2$ мм, $h=4,3$ мм, извлеченный из магнитного штифта прибора Osstell ISQ, закреплялся с помощью клея (типа эпоксидной смолы) прямо в отверстии для заглушки на уровень (заподлицо) с верхней кромкой. Во втором случае нижняя часть штифта (SmartPeg) обрезалась и на оставшейся части нарезалась соответствующая резьба так, чтобы после крепления магнита над поверхностью имплантата выступала его часть высотой 2 мм, за которую можно было завинчивать и отвинчивать эту заглушку. Результаты измерения КСИ ${ }_{b}$ для таких заглушек приведены на рис. 4. Глубина крепления $l$ (завинчивания имплантата в аналог костной ткани) изменялась в процессе эксперимента, т.е. после проведения измерения для некоторого значения $l$ имплантат завинчивался глубже, проводилось измерение $\mathrm{KCИ}_{b}$ для нового значения $l$ и т.д. При этом использовались имплантаты фирмы Conmet диаметром 4 мм и длиной 15 и 19 мм. Поскольку результаты измерения КСИ ${ }_{b}$ не зависели от размеров имплантатов и способа крепления магнита (вида 
магнитной заглушки), на графике (см. рис. 4) указана только величина площади $S$ контакта имплантата с материалом. В части экспериментов измерение КСИ проводилось через слой свиной кожи толщиной 1-2 мм. Эти экспериментальные точки обозначены кружочками. Другая часть опытных данных, изображенная на графике треугольниками, соответствует измерениям без покрытия (сплошная линия результаты обработки опытных данных методом наименьших квадратов).

Результаты измерений показывают, что слой свиной кожи, как и некоторые другие немагнитные материалы (органическое стекло, полиэтилен, линолеум), не влияет на показания прибора.

Таким образом, при наличии магнитной заглушки можно проводить мониторинг КСИ ${ }_{b}$, не вскрывая слизистый лоскут, т.е. не оказывая влияния на процесс остеоинтеграции. Эта методика может быть особенно полезной при оценке результатов различных курсов интенсивной терапии, когда ожидаются быстрые изменения прочности крепления дентальных имплантатов и, конечно, при определении сроков готовности имплантатов к функциональной нагрузке.

Одной из важнейших характеристик имплантатов является их несущая способность. Ее оценка является необходимой и очень важной частью предоперационного анализа. Информация по этому вопросу в литературе отсутствует, и выбор числа и размеров Mini Dental Implants основывается на опыте и интуиции лечащего врача. Имеется множество публикаций, в которых обсуждаются критерии готовности имплантатов к функциональным нагрузкам. Например, используя прибор Osstell ISQ, большинство авторов считает, что при значении коэффициента стабильности КСИ $\geq 65$ имплантат готов к функциональным нагрузкам $[3,7]$. Но не указывается, как определить величину этих нагрузок. Отметим лишь некоторые общие соображения. Если после установки дентального имплантата коэффициент стабильности растет (или, по крайней мере, не убывает) и составляет КСИ = 65 (за исключением первых трех недель, когда его значение может сначала убывать, а затем расти до первоначального уровня), то ситуацию можно считать удовлетворительной. Если при этом известен рацион питания пациента (т.е. количество и твердость потребляемых продуктов), можно оценить величину соответствующих жевательных нагрузок и по методике, изложенной в работе [8], попытаться оценить несущую способность этих дентальных имплантатов. Не имея таких статистических данных, сравним несущую способность Mini Dental Implants с несущей способностью классических винтовых имплантатов, принимая ее за основу. Для этого можно предложить следующий критерий: два имплантата, имеющие одинаковые коэффициенты продольной жесткости (или стабильности), эквивалентны, т.е. с механической точки зрения обладают одинаковой несущей способностью.

На рис. 5 приведена зависимость коэффициента продольной жесткости $K_{b}$

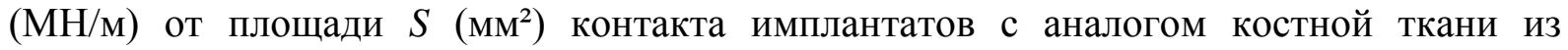
полиуретана. Эта зависимость близка к линейной.

Эксперименты показывают, что коэффициенты продольной жесткости больших Mini Dental Implants мало отличаются от аналогичных значений $K_{b}$ для классических имплантатов небольших размеров и хорошо вписываются в общую закономерность. Так как последние используются при восстановительных операциях как несущие элементы протеза, то нет оснований считать, что для этой же цели нельзя применять мини-имплантаты. В действительности врачам-ортопедам уже давно приходится использовать Mini Dental Implants как несущие элементы при реставрациях в ряде особых случаев (когда эстетическая функция становится важнее жевательной или при настоятельных просьбах пациентов). В этих случаях при выборе числа и размеров Mini Dental Implants желательно иметь научно обоснованные рекомендации. 


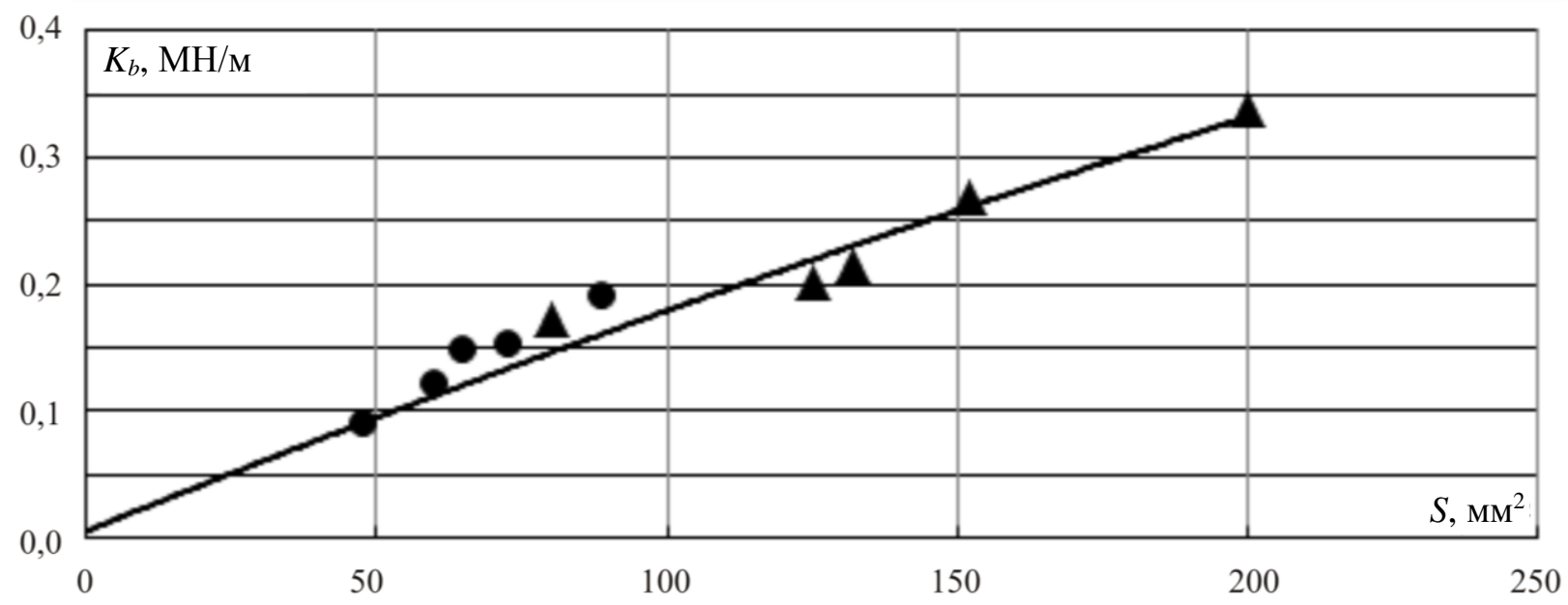

Рис. 5. Зависимость коэффициентов продольной жесткости имплантатов $K_{b}$ от площади $S$ их контакта с костной тканью: $\boldsymbol{\Delta}$ - классические винтовые имплантаты;

• - мини-имплантаты

Зависимость, приведенная на рис. 5, может помочь в решении этого вопроса. Действительно, вместо одного классического дентального имплантата можно, например, установить два Mini Dental Implants, если их несущие способности эквивалентны (т.е. если $k=k_{1}+k_{2}$ ), но их установка возможна за одно посещение при минимальном хирургическом вмешательстве и последующем «немедленном» нагружении. Следует подчеркнуть, что, хотя эксперименты, проведенные на аналогах костной ткани, не дают представления о реальных значениях коэффициентов продольной жесткости, они позволяют сравнить между собой несущие способности имплантатов различных размеров и формы.

\section{СРАВНЕНИЕ КОЭФФИЦИЕНТОВ ПРОДОЛЬНОЙ СТАБИЛЬНОСТИ И ПРОДОЛЬНОЙ ЖЕСТКОСТИ}

Как отмечалось выше, прочность крепления дентальных имплантатов в костной ткани можно определять двумя способами: измеряя коэффициенты стабильности $\left(К С И_{b}\right)$ с помощью прибора Osstell ISQ (резонансно-частотный анализ) и определяя коэффициенты жесткости $\left(K_{b}\right)$ по методике лазерного тестирования (статические испытания). Очевидно, что каждому дентальному имплантату, закрепленному в какомлибо аналоге костной ткани, соответствуют два коэффициента $\left(\mathrm{KCИ}_{b}\right.$ и $\left.K_{b}\right)$, которые характеризуют прочность его крепления в данном материале. Опытные данные по


форме и размерам дентальных имплантатов, закрепленных в разных аналогах костной ткани, очевидно, образуют некоторое множество $\left\{\mathrm{KCИ}_{b}, K_{b}\right\}$. Возникает вопрос, является ли это множество набором случайных величин или оно отражает определенную физическую закономерность? На рис. 6 изображены опытные данные для всех 10 исследованных дентальных имплантатов, закрепленных в различных аналогах костной ткани. Опытные данные приведены в табл. 1-3.

Приведенные результаты показывают, что существует взаимно однозначное соответствие между этими величинами. Разброс опытных данных, по-видимому, является следствием не только погрешности измерений. Коэффициенты стабильности, очевидно, зависят не только от коэффициентов жесткости, но и от других параметров (плотности среды, модуля Юнга, коэффициента Пуассона, вязкости костной ткани и др.). 
Таблица 1

Коэффициенты продольной жесткости и продольной стабильности (твердый пенопласт)

\begin{tabular}{|c|c|c|c|c|c|c|c|c|c|c|}
\hline \multirow{2}{*}{ Параметр } & \multicolumn{10}{|c|}{ Номер образца } \\
\cline { 2 - 12 } & 1 & 2 & 3 & 4 & 5 & 6 & 7 & 8 & 9 & 10 \\
\hline$S$, мм $^{2}$ & 79 & 85 & 120 & 123 & 124 & 148 & 150 & 200 & 200 & 210 \\
\hline$K_{b}, \mathrm{MH} / \mathrm{M}$ & 0,60 & 0,73 & 1,07 & 1,02 & 1,11 & 1,13 & 1,27 & 1,63 & 1,72 & 1,89 \\
\hline КСИ $_{b}$ & 69 & 73 & 74 & 74 & 75 & 76 & 77 & 79 & 80 & 81 \\
\hline
\end{tabular}

Таблица 2

Коэффициенты продольной жесткости и продольной стабильности (полиуретан, 1-5 -классические дентальные имплантаты, 6-10 - Mini Dental Implants)

\begin{tabular}{|c|c|c|c|c|c|c|c|c|c|c|}
\hline \multirow{2}{*}{ Параметр } & \multicolumn{10}{|c|}{ Номер образца } \\
\cline { 2 - 12 } & 1 & 2 & 3 & 4 & 5 & 6 & 7 & 8 & 9 & 10 \\
\hline$S$, мм $^{2}$ & 78 & 119 & 126 & 143 & 190 & 47 & 58 & 64 & 105 & 106 \\
\hline$K_{b}, \mathrm{MH} / \mathrm{M}$ & 0,135 & 0,200 & 0,205 & 0,240 & 0,330 & 0,107 & 0,117 & 0,127 & 0,182 & 0,181 \\
\hline $\mathrm{KCИ}_{b}$ & 25 & 32 & 32 & 37 & 41 & 35 & 35 & 35 & 40 & 35 \\
\hline
\end{tabular}

Коэффициенты продольной жесткости и продольной стабильности

(1-5 - липа, классические дентальные имплантаты, 6-10 - боксил, классические дентальные имплантаты)

\begin{tabular}{|c|c|c|c|c|c|c|c|c|c|c|}
\hline \multirow{2}{*}{ Параметр } & \multicolumn{10}{|c|}{ Номер образца } \\
\cline { 2 - 12 } & 1 & 2 & 3 & 4 & 5 & 6 & 7 & 8 & 9 & 10 \\
\hline$S$, мм $^{2}$ & 85 & 125 & 132 & 150 & 200 & 84 & 118 & 143 & 186 & 189 \\
\hline$K_{b}, \mathrm{MH} / \mathrm{M}$ & 1,320 & 2,000 & 2,120 & 2,570 & 2,560 & 0,019 & 0,014 & 0,022 & 0,011 & 0,027 \\
\hline $\mathrm{KCИ}_{b}$ & 77 & 83 & 83 & 85 & 81 & 2 & 3 & 5 & 1 & 7 \\
\hline
\end{tabular}

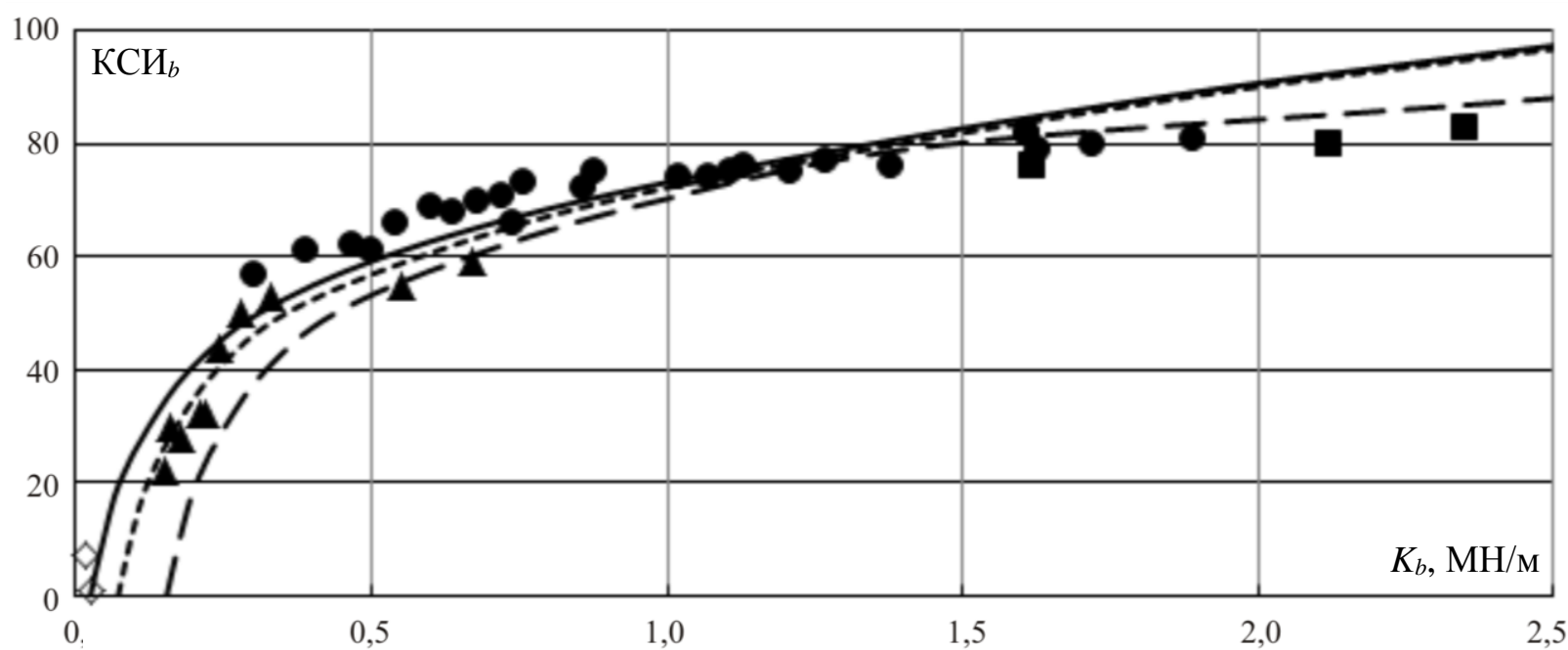

Рис. 6. Зависимость коэффициентов продольной стабильности КСИ от коэффициентов продольной жесткости $K_{b}$ : • - твердый пенопласт; полиуретан; - - липа; $\diamond-$ боксил 
Для того чтобы понять физический смысл закономерности, изображенной на рис. 6, построим простейшую теоретическую модель продольных колебаний дентального имплантата, закрепленного в вязкоупругой среде. Пусть $m$ - масса дентального имплантата вместе с магнитной заглушкой (или измерительным колпачком с магнитом), $x$ - его перемещение в продольном направлении. В предыдущих работах при расчете напряженно-деформированного состояния и оценке величины резонансной частоты предполагалось, что для костной ткани и ее аналогов справедлив закон Гука [6]. В действительности это можно рассматривать лишь как первое приближение, когда вязкие (диссипативные) силы, зависящие от скорости нагружения, малы по сравнению с упругими. Рассмотрим теперь видоизменение закона Гука, предложенное Кельвином и Фойгтом, когда связь между модулем Юнга $E$ и относительной деформацией $\varepsilon$ имеет вид

$$
\sigma=E \varepsilon+\eta \frac{d \varepsilon}{d t}
$$

где $\sigma$ - напряжение; $\eta$ - вязкость материала; $t$ - время [2].

Первый член выражения для $\sigma$ является упругой составляющей напряжения, второй - его диссипативной частью.

Для дентального имплантата, закрепленного в таком аналоге костной ткани, уравнение продольных вынужденных колебаний имеет вид

$$
m \ddot{x}=-k_{b} x-\mu \dot{x}+Q_{0} \sin p t
$$

или

$$
\ddot{x}+2 b \dot{x}+k^{2} x=P_{0} \sin p t,
$$

где $2 b=\mu / m, k^{2}=k_{b} / m, P_{0}=Q_{0} / m$ ( $\mu$ - вязкость материала; $m$ - масса дентального имплантата; $Q_{0}$ - амплитуда и $p$ - частота возмущающей (вынуждающей) силы). Как известно, в этом случае амплитуда $A$ вынужденных колебаний [9]

$$
A=\frac{P_{0}}{\sqrt{\left(k^{2}-p^{2}\right)^{2}+4 b^{2} p^{2}}} .
$$

Нетрудно показать, что при $p=\sqrt{k^{2}-2 b^{2}}$ амплитуда вынужденных колебаний имеет максимум. При $k^{2} \leq 2 b^{2}$ вязкость костной ткани выше критического значения и амплитуда колебаний $A$ монотонно убывает во всем диапазоне изменения параметра $p$.

Определим теперь коэффициенты вязкости аналогов костной ткани. Собственная частота упругих колебаний системы «дентальный имплантат + магнитная заглушка», закрепленной в аналоге костной ткани, равна $k=\sqrt{k_{b} / m}$. Резонансная частота данной системы, закрепленной в вязкоупругой среде, в соответствии с результатами измерения КСЙ с помощью прибора Osstell ISQ, равна $p=2 \pi f($ КСИ).

Зависимость между резонансной частотой $f$ вынуждающей силы и коэффициентом КСИ стабильности для дентального имплантата + SmartPeg имеет вид

$$
\text { КСИ }=-2,4 \cdot 10^{-14} \cdot f^{4}+7,1 \cdot 10^{-10} \cdot f^{3}-7,8 \cdot 10^{-6} \cdot f^{2}-4,45 \cdot 10^{-2} \cdot f-37,
$$

см. также табл. 4, эти данные предоставлены руководством фирмы Osstell AB.

Таким образом, коэффициент вязкости среды можно определить по формуле $b=0,707 \sqrt{k^{2}-p^{2}}$. Полученные значения параметров $b$, определенные для твердого пенопласта, полиуретана и липы при обработке опытных данных, соответственно, равны: $b_{1}=(9,2 \pm 3,5) 10^{3} \mathrm{c}^{-1}$ (твердый пенопласт), $b_{2}=(9,4 \pm 2,1) 10^{3} \mathrm{c}^{-1}$ (полиуретан) и 
Зависимость коэффициента стабильности КСИ от резонансной частоты $f$

\begin{tabular}{|c|c|c|c|c|c|c|c|c|c|c|c|}
\hline \multirow{2}{*}{ Параметр } & \multicolumn{10}{c|}{ КСИ } \\
\cline { 2 - 13 } & 0 & 10 & 20 & 30 & 40 & 50 & 60 & 70 & 80 & 90 & 99 \\
\hline$f$, Гц & 977 & 1343 & 1709 & 2197 & 2747 & 3479 & 4517 & 5737 & 7263 & 8728 & 10132 \\
\hline
\end{tabular}

Таблииа 5

Коэффициенты стабильности и коэффициенты жесткости дентальных имплантатов, установленных в аналогах костной ткани (расчет)

\begin{tabular}{|c|c|c|c|c|c|c|c|c|c|c|c|}
\hline \multirow{2}{*}{$K_{b}, \mathrm{MH} / \mathrm{M}$} & \multicolumn{10}{|c|}{$\mathrm{KCИ}_{b}$} \\
\cline { 2 - 12 } & 0 & 10 & 20 & 30 & 40 & 50 & 60 & 70 & 80 & 90 & 99 \\
\hline$b=0$ & 0,024 & 0,046 & 0,073 & 0,123 & 0,192 & 0,308 & 0,519 & 0,855 & 1,340 & 1,940 & 2,610 \\
\hline$b=6 \cdot 10^{3} \mathrm{c}^{-1}$ & 0,071 & 0,092 & 0,121 & 0,169 & 0,238 & 0,354 & 0,579 & 0,901 & 1,388 & 1,980 & 2,660 \\
\hline$b=10^{4} \mathrm{c}^{-1}$ & 0,153 & 0,175 & 0,203 & 0,252 & 0,321 & 0,437 & 0,662 & 0,984 & 1,471 & 2,660 & 2,740 \\
\hline
\end{tabular}

$b_{3}=(19,2 \pm 2,3) 10^{3} \mathrm{c}^{-1}$ (липа). Коэффициенты жесткости $K_{b}$ и стабильности КСИ $\mathrm{K}_{b}$ этих материалов, использованные при расчетах, приведены в табл. 1-3. Зная величины параметров $b$ этих материалов, получаем следующие средние значения их коэффициентов вязкости: твердый пенопласт - $\mu=(11,9 \pm 4,5) \mathrm{H} /(\mathrm{м} / \mathrm{c})$; полиуретан $\mu=(12,1 \pm 2,7) \mathrm{H} /(\mathrm{м} / \mathrm{c})$; липа $-\mu=(24,8 \pm 3,0) \mathrm{H} /(\mathrm{m} / \mathrm{c})$.

Для боксила определить значение параметра $b$ и, следовательно, коэффициент вязкости $\mu$ не удалось из-за большого разброса опытных данных, хотя, очевидно, что для него значение коэффициента вязкости является малым $(\mu \approx 1 \mathrm{H} /(\mathrm{м} / \mathrm{c}))$.

Исследования по определению коэффициентов вязкости $\mu$, приведенные выше, надо рассматривать как методические, а значения параметров $b$ и $\mu$ как приближенные (оценочные).

Пользуясь этим подходом, можно было бы определить вязкость костной ткани, в которой крепятся имплантаты. Но, к сожалению, информация о коэффициентах стабильности КСИ ${ }_{b}$ и жесткости $K_{b}$ дентальных имплантатов, закрепленных в костной ткани, отсутствует. Попытаемся оценить достоверность предложенной выше теоретической модели. Предположим, что параметры $b$ аналогов костной ткани, в которых крепятся дентальные имплантаты, являются некоторыми константами. Если теоретическая модель справедлива, расчетные кривые, полученные по формулам для вязкоупругой среды, должны удовлетворительно согласовываться с опытными данными.

Алгоритм расчета этих зависимостей следующий:

- Задаем параметр $b$ и значение коэффициента КСИ $b$ продольной стабильности.

- Определяем соответствующую КСИ $b$ резонансную частоту $f$ (см. табл. 4 или формулу КСИ = КСИ $(f))$.

- Вычисляем значение круговой частоты $p=2 \pi f$.

- Определяем величину коэффициента продольной жесткости по формуле $K_{b}=k^{2} m$, где $k^{2}=p^{2}+2 b^{2}, m$ - масса системы «дентальный имплантат + магнитный колпачок».

Пример:

$b=10^{4} \mathrm{c}^{-1}$, КСИ $=60, f=4517 \mathrm{c}^{-1}, p=2 \pi f=28,37 \cdot 10^{3} \mathrm{c}^{-1}$;

$m=0,52+0,125=0,645 \Gamma=0,645 \cdot 10^{-3}$ кг (дентальный имплантат № $4+$ магнитная заглушка); 


$$
\begin{aligned}
& b=10,0 \cdot 10^{3} \mathrm{c}^{-1} ; \\
& K_{b}=k^{2} m=\left(p^{2}+2 b^{2}\right) m=\left(28,37^{2}+200\right) 10^{6} \cdot 0,645 \cdot 10^{-3}=0,648 \cdot 10^{6} \mathrm{H} / \mathrm{M} .
\end{aligned}
$$

На рис. 6, приведенном выше, сплошной линией, линиями с коротким и длинным штрихом изображены расчетные кривые, полученные при использовании предложенного выше алгоритма расчета для системы «дентальный имплантат № 4 + магнитная заглушка», установленной в вязкоупругой среде, при значениях параметров $b$, равных $b_{1}=0, b_{2}=6,0 \cdot 10^{3} \mathrm{c}^{-1}, b_{3}=10^{4} \mathrm{c}^{-1}$ (см. табл. 5).

Сравнение показывает, что расчетные кривые для упругой $(b=0)$ и двух вязкоупругих $\left(b=6 \cdot 10^{3} \mathrm{c}^{-1}\right.$ и $\left.b=10 \cdot 10^{3} \mathrm{c}^{-1}\right)$ сред хорошо качественно и неплохо количественно соответствуют опытным данным, полученным при использовании аналогов костной ткани. При $50<$ КСИ $_{b}<85$ отличие составляет 8-12\%. При $0<\mathrm{KCИ}_{b}<50$ влияние вязкости становится существенным, особенно при малых

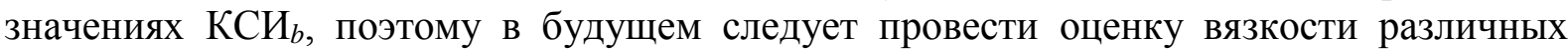
типов костной ткани. Однако в основном это необходимо для мини-имплантатов и классических дентальных имплантатов малых размеров, поскольку при таких значениях $\mathrm{KCИ}_{b}$ средние и большие классические имплантаты обычно подлежат удалению.

\section{ЗАКЛЮЧЕНИЕ}

Полученные результаты подтверждают адекватность прибора Osstell ISQ и целесообразность использования коэффициентов продольной стабильности для оценки готовности дентальных имплантатов к функциональным нагрузкам. Использование магнитных заглушек и магнитных колпачков позволит значительно расширить возможности неинвазивного контроля уровня остеоинтеграции и повысить эффективность реконструктивной хирургии и ортопедии.

Проведенные исследования подтверждают, что костную ткань верхней и нижней челюстей можно рассматривать как вязкоупругую среду и позволяют определять вязкость костной ткани.

\section{СПИСОК ЛИТЕРАТУРЫ}

1. Арутюнов С.Д., Ерошин В.А., Степанов А.Г., Бойко А.В., Даов А.Н., Мацаберидзе Л.Г. Новые тенденции использования дентальных мини-имплантатов // Российский вестник дентальной имплантологии. - 2013. - Т. 17, № 2. - С. 14-20.

2. Дейвис Р.М. Волны напряжений в твердых телах. - М.: Изд-во иностр. лит-ры, 1961. - 103 с.

3. Дробышев А.Ю., Дронов М.В. Оценка стабильности и остеоинтеграции дентальных имплантатов с применением резонансно-частотного метода // Институт стоматологии. - 2007. - № 1. - С. 128-129; 2007. - № 3. - С. 64-65.

4. Ерошин В.А., Арутюнов С.Д., Арутюнов А.С., Унанян В.Е., Бойко А.В. Подвижность дентальных имплантатов: приборы и методы диагностики // Российский журнал биомеханики. - 2009. - Т. 13, № 2. - С. 34-48.

5. Ерошин В.А., Джалалова М.В., Бойко А.В., Арутюнов С.Д., Степанов А.Г., Даов А.Н. Подвижность дентальных имплантатов: новые возможности известного прибора // Российский журнал биомеханики. - 2015. - Т. 19, № 3. -С. 273-281.

6. Ерошин В.А., Джалалова М.В. Напряженно-деформированное состояние биомеханической системы имплантат - упругое основание // Российский журнал биомеханики. - 2012. - Т. 16, № 3. - С. 82-95.

7. Маркин В.А., Олесова В.Н., Арутюнов С.Д., Мушаев И.Ч., Поздняев А.И., Тарафутдинов Д.М. Частотно-резонансное тестирование внутрикостных имплантатов на нижней челюсти как метод обследования их непосредственной нагрузки // Российский стоматологический журнал. - 2006. T. 10, № 1. - C. 44-46.

8. Перевезенцева А.А. Врачебная тактика выбора конструкционного материала временных протезов: дис. ... канд. мед. наук. - М., 2012. - 134 с.

9. Тарг С.М. Краткий курс теоретической механики. - М.: Наука, 1968. - 479 с. 
10. Balkin B.E., Steflik D.E., Naval F. Mini-dentasi implant insertion with the auto-advance technique for ongoing applications // Journal of Oral Implantology. - 2001. - Vol. 27. - P. 32-37.

11. Bulard R.A. Mini dental implants: enhancing patient satisfaction and practice income // Dent. Today. 2001. - Vol. 20. - P. 1-5.

12. Christensen G.J. The "mini implant" has arrived // Journal of American Dental Association. - 2006. Vol. 137. - P. 387-390.

13. Christensen G.J. Implants and the general practitioner // JADA. - 2000. - Vol. 131. - P. 359-361.

14. Flanagan D., Mascolo A. The mini dental implant in fixed and removable prosthetics: a review // Journal of Oral Implantology. - 2011. - Vol. 37. - P. 123-132.

15. Shatkin T.E., Shatkin S., Oppenheimer B.D., Oppenheimer A.J. Mini dental implants for long term fixed and removable prosthetics: a retrospective analysis of 2514 implants placed over a five year period // Compendium. - 2007. - Vol. 28. - P. 36-41.

\title{
QUOTIENTS OF LONGITUDINAL STABILITY FOR DENTAL IMPLANTS
}

\author{
V.A. Yeroshin, M.V. Dzhalalova, A.V. Boyko, \\ S.D. Arutyunov, A.G. Stepanov (Moscow, Russia)
}

A concept of longitudinal stability is introduced for dental implants. It is well known that the Osstell ISQ device (Implant Stability Quotient, Sweden), along with forced oscillations of dental implants arising under lateral loads, may also excite longitudinal oscillations and register their resonance frequency. The objective of the study is to substantiate possibility to apply quotients of longitudinal stability in order to assess readiness of dental implants for functional loads, as well as to verify methods of their measurement using the Osstell ISQ device by comparison with appropriate quotients of longitudinal rigidity measured by laser testing. In comparison with the traditional technique, the proposed one has a number of advantages and enables creation of a new and more perfect base for noninvasive monitoring of dental implant osteointegration process. For example, the use of modified "magnetic plugs" instead of magnetic pins (SmartPeg) allows quotients of longitudinal stability to be measured through a mucous membrane of alveolar bone, i.e. a dental implant will not be open for further use as a fixed denture support, and modified "magnetic caps" allow determination of quotients of longitudinal stability for spherical headed mini implants used for additional fixation of detachable dentures when teeth are absent totally. Please note that the traditional Osstell ISQ technique does not enable measurements of coefficients of stability for the said mini implants. Introduction of quotients of longitudinal stability has made it possible to create a simple theoretical model of oscillations for dental implants fixed in elastic and viscous-elastic medium, to relate quotients of longitudinal stability and longitudinal rigidity to each other, and to assess bearing capacity of dental implants. Moreover, recommendations remain the same when assessing implant readiness for functional loads using quotients of longitudinal stability since the ratio of quotients of stability for longitudinal and lateral loads approximates unity.

Key words: prosthetic dentistry, dental implant, mini dental implant, quotient of stability, quotient of rigidity.

Получено 05 сентября 2016 